\title{
Transgenic male zebrafish just can't compete
}

Genetically modified organisms (GMOs) possess novel and, often, heritable genotypes that typically would not occur without human intervention. Transgenic modifications can alter the fitness of a species by enhancing or diminishing its reproductive success and survivability. These consequences can be managed in a laboratory setting, but if GMOs are introduced into uncontrolled environments, they might pose a threat to populations and ecosystems of their unmodified conspecifics. Some GMOs have already been released into uncontrolled environments, and others are closely regulated but still at risk of escape or accidental release. It is therefore important to understand how the fitness of GMOs influences their evolutionary outcomes when introduced to a natural population.

Researchers at Purdue University (West Lafayette, IN) simulated this scenario in experimental populations using wild-type and transgenic populations of zebrafish (Evolution 69, 1143-1157; 2015). By intermating transgenic and wild-type zebrafish over 11 generations, the researchers produced a transgenic population that was similar to the wild-type population in its genetic background and fitness components, except that it fluoresced red and had lower mating success.

Mating success can be influenced by multiple factors that include intersexual mate choice and intrasexual mate competition. In this case, red fluorescence seems to appeal to female zebrafish but behavioral observations found that wild-type males usually outcompeted the transgenic males by behaving more aggressively toward both sexes.

The researchers also carried out a longterm study of 18 populations for up to 15 generations to understand how the populations would evolve after the introduction of the red fluorescence transgene. Transgenic and wild-type zebrafish had similar mortality, but by the 15th generation all but one population had lost the transgene. "The females didn't get to choose," said author William Muir in a press release. "The wild-type males drove away the reds and got all the mates. That's what drove the transgene to extinction."

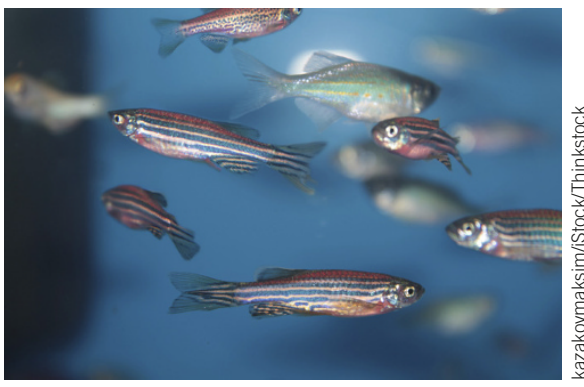

These findings suggest that the transgenic trait of red fluorescence would not persist in a natural population. To accompany these findings, Muir and his peers applied a demographic model that simulates evolution within a population based on estimations of fitness. This model closely predicted the decline and extinction of the transgenic trait, indicating that it might be a valuable tool for assessing the risk of genetic pollution from uncontrolled GMOs. Muir emphasized that the model does not make any specific recommendations on whether to release GMOs. "It simply says what would likely happen if we did."

Gregory D. Larsen

\section{TEACHING OLD DRUGS NEW TRICKS}

For several years, the biomedical research community has recognized that compounds already approved for human use might have additional therapeutic applications. To facilitate investigation of new applications for existing drugs, the US National Institutes of Health (NIH) assembled several collections of molecules that can be screened for specific characteristics. Two recent papers report the screening of these collections together with animal testing to identify promising new uses for approved drugs.

T. Jake Liang (NIH, Bethesda, MD) and colleagues screened the NIH Chemical Genomics Center Pharmaceutical Collection to detect compounds with activity against hepatitis C virus (HCV). HCV infection can become chronic, leading to liver disease, cirrhosis and cancer. Currently available treatments are costly and have unwanted side effects. "Although hepatitis $\mathrm{C}$ is curable, there is an unmet need for effective and affordable medication," Liang told NIH Research Matters. His research group discovered antiviral activity in chlorcyclizine $\mathrm{HCl}(\mathrm{CCZ})$, an over-the-counter antihistamine drug approved for the treatment of allergy symptoms. CCZ inhibited HCV infection in human liver cells in vitro. CCZ also limited HCV infection in mice with engrafted human liver cells for 4-6 weeks without eliciting drug resistance. " $\mathrm{CCZ}$ represents a promising candidate for drug repurposing and further development as an effective and accessible agent for treatment of HCV infection," the group concluded (Sci. Transl. Med. 7, 282ra49; 2015).

Robert H. Miller (George Washington University, Washington, DC), Paul J. Tesar (Case Western Reserve University, Cleveland, OH) and collaborators searched a separate library called the NIH Clinical Collections for drugs able to trigger maturation of oligodendrocyte progenitor cells (OPCs) into oligodendrocytes, which are responsible for myelination of nerve cells. This maturation process is faulty in multiple sclerosis (MS), resulting in reduced myelination and chronic disability. The researchers identified two compounds, miconazole (an antifungal) and clobetasol (a steroid), that stimulated OPC maturation and remyelination. Both drugs improved remyelination in a mouse model of focal demyelination and promoted precocious myelination in the absence of disease in young mouse pups. The drugs also induced differentiation of human OPCs into oligodendrocytes. Clobetasol but not miconazole reduced disease severity in an immune-mediated mouse model of MS, and both compounds were effective in a chronic mouse model of MS, with nearly all the treated animals regaining use of at least one hind limb (Nature doi:10.1038/nature14335; published online 20 April 2015). The scientists believe that "these drugs... could advance into clinical trials for the currently untreatable chronic progressive phase of MS." 